\title{
Retrograde Cerebral Venous Air Embolism on Susceptibility-Weighted Imaging
}

\author{
Yunsup Hwang, Young-Joo Kim
}

Keywords: Cerebral air embolism, Magnetic resonance (MR), Susceptibility-weighted image (SWI), Retrograde cerebral venous air embolism

A 71-year-old man with a history of diabetes underwent a central venous cannulation of the right internal jugular vein for treatment. At 6 hours after catheterization, while sitting upright in bed, he suddenly displayed a decrease in mental capacity after violent coughing with a central venous catheter disconnected. Non-enhanced brain CT (Figure 1A) showed extensive serpiginous air bubbles in the sulci of the right convexity and collections of gas in the venous sinuses. A few locules of air were also noted in the right frontal and parietal lobes. Susceptibility-weighted MR imaging (SWI) (Figure 1B) revealed multifocal serpentine dark signal intensities distending the right frontal-parietal sulci and in the superior sagittal sinus,
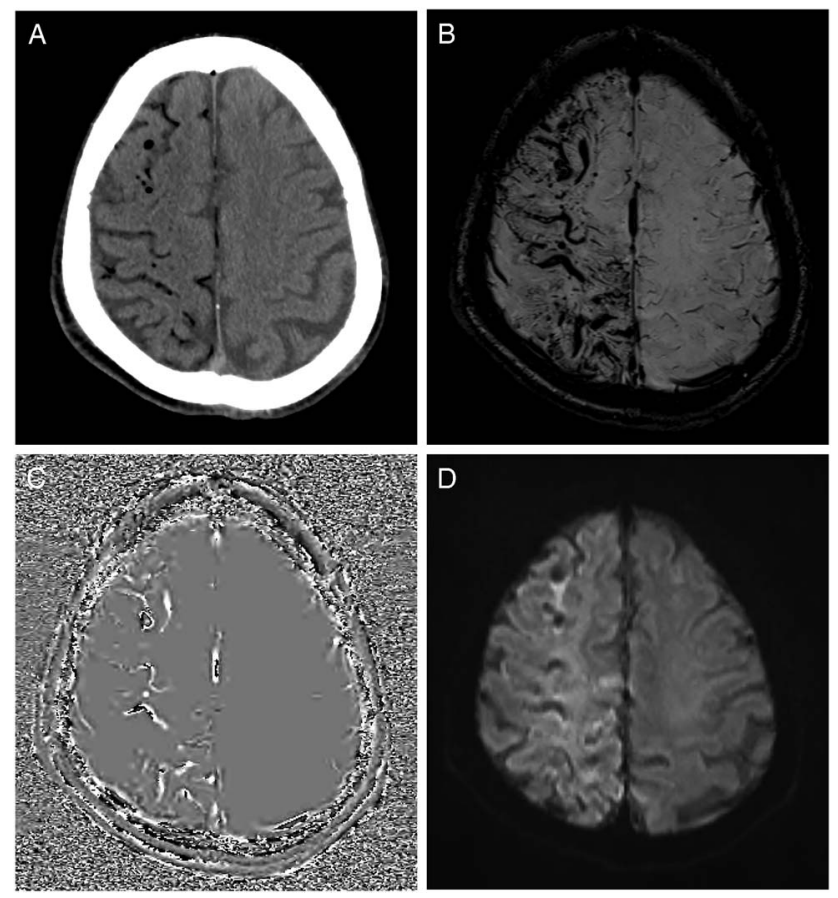

Figure 1: Axial non-enhanced brain CT (A) demonstrates serpentine and tubular air densities in the right frontal-parietal sulci and venous sinuses, and multifocal small air bubbles in the right frontal and parietal lobes. Axial susceptibility-weighted MR imaging (SWI) (B) and phase image $(C)$ show prominent dark signal intensities with blooming and aliasing artifacts, which corresponds with air emboli on nonenhanced CT. The diffusion-weighted image $(D)$ shows diffuse areas of restricted diffusion in the right cerebral hemisphere. and additional ovoid dark signal intensities in the right frontal and parietal lobes. Phase image (Figure 1C) showed mixed high and low signal intensities in the corresponding locations, owing to an aliasing artifact from air. Diffusion MRI revealed extensive restricted diffusion in the right cerebral hemisphere (Figure 1D). The patient remained comatose despite hyperbaric oxygen therapy, and was discharged to a suitable nursing-home accommodation.

On the basis of the observed entry of air into the right hemisphere, air influx from the disconnected central venous catheter seemed to be the cause of the cerebral venous air embolism. Retrograde cerebral venous air embolism associated with central venous catheterization often goes undetected and can cause major morbidity and mortality. ${ }^{1}$ Diagnosis of cerebral air embolism can be easily confirmed using brain CT because air bubbles show a specific low attenuation. On MRI, air appears dark signal intensity on all conventional MRI sequences. However, depending on the location and morphology, air bubbles in the intracranial venous system can be mistaken for blood product or cortical veins. ${ }^{2}$ We found that air bubbles in the cortical veins showed engorged tubular dark signal intensities in the sulci with blooming and aliasing artifacts on SWI owing to marked susceptibility artifact caused by gas. On the other hand, subarachnoid hemorrhage has irregular nonuniform dark signal intensity with a rough boundary and a slightly triangular form. ${ }^{3}$ Image findings of acute cortical vein thrombosis can be similar to those of cerebral venous air embolism on SWI owing to the increased concentration of deoxyhemoglobin in the involved veins. ${ }^{4}$ In this case, clinical history and immediate CT scan are helpful for differential diagnosis. In conclusion, if there are serpentine dark signal intensities with a smooth margin and blooming on SWI, with heterogeneous aliasing pattern on the phase image, retrograde cerebral venous air embolism should be considered in patients with neurological deterioration and central venous catheterization.

From the Department of Radiology, Uijeongbu St. Mary's Hospital, College of Medicine, The Catholic University of Korea, Gyeonggi-do, Korea (YH,Y-JK).

Received February 26, 2017. Revisions Submitted February 28, 2018. Date of ACCEPTANCE March 16, 2018.

Correspondence to: Y.-J. Kim, Department of Radiology, Uijeongbu St. Mary's

Hospital, College of Medicine, The Catholic University of Korea, Uijeongbu St. Mary's Hospital, 65-1 Geumo-dong, Uijeongbu-si, Gyeonggi-do, 480-717, Korea.

Email: violet2@ catholic.ac.kr 


\section{Statement OF AUTHORShiP}

YH and Y-JK drafted and revised the manuscript for intellectual content and created the figure together.

\section{Disclosures}

YH and Y-JK have no disclosures.

\section{REFERENCES}

1. Schlimp CJ, Loimer T, Rieger M, Schmidts MB, Lederer W. Pathophysiological mechanism and immediate treatment of retrograde cerebral venous air embolism. Intensive Care Med. 2006;32:945.

2. Bosemani T, Verschuuren SI, Poretti A, Huisman TA. Pitfalls in susceptibility-weighted imaging of the pediatric brain. J Neuroimaging. 2014;24:221-5.

3. Wu Z, Li S, Lei J, An D, Haacke EM. Evaluation of traumatic subarachnoid hemorrhage using susceptibility-weighted imaging. AJNR Am J Neuroradiol. 2010;31:1302-10.

4. Boukobza M, Crassard I, Bousser MG, Chabriat H. MR imaging features of isolated cortical vein thrombosis: diagnosis and follow-up. AJNR Am J Neuroradiol. 2009;30:344-8. 